\title{
Rationale, design and conduct of a randomised controlled trial evaluating a primary care-based complex intervention to improve the quality of life of heart failure patients: HICMan (Heidelberg Integrated Case Management)
}

\author{
Frank Peters-Klimm*1, Thomas Müller-Tasch², Dieter Schellberg2, \\ Jochen Gensichen ${ }^{3}$, Christiane Muth ${ }^{3}$, Wolfgang Herzog ${ }^{2}$ and \\ Joachim Szecsenyi ${ }^{1}$
}

Address: ${ }^{1}$ Department of General Practice and Health Services Research, University Hospital of Heidelberg, Voßstraße 2, 69115 Heidelberg, Germany, ${ }^{2}$ Department of Psychosomatic and General Internal Medicine, University of Heidelberg Hospital, Germany and ${ }^{3}$ Institute for General Practice, Chronic Care and Health Services Research University of Frankfurt, Theodor-Stern-Kai 7, 60590 Frankfurt a. M., Germany

Email: Frank Peters-Klimm* - Frank.Peters@med.uni-heidelberg.de; Thomas Müller-Tasch - Thomas.Mueller-Tasch@med.uni-heidelberg.de; Dieter Schellberg - Dieter.Schellberg@med.uni-heidelberg.de; Jochen Gensichen - Gensichen@allgemeinmedizin.uni-frankfurt.de; Christiane Muth - Muth@allgemeinmedizin.uni-frankfurt.de; Wolfgang Herzog - Wolfgang.Herzog@med.uni-heidelberg.de; Joachim Szecsenyi - Joachim.Szecsenyi@med.uni-heidelberg.de

* Corresponding author

\section{Published: 23 August 2007}

BMC Cardiovascular Disorders 2007, 7:25 doi:10.1 186/147/-226I-7-25

This article is available from: http://www.biomedcentral.com/|47|-226I/7/25

(C) 2007 Peters-Klimm et al; licensee BioMed Central Ltd.

This is an Open Access article distributed under the terms of the Creative Commons Attribution License (http://creativecommons.org/licenses/by/2.0), which permits unrestricted use, distribution, and reproduction in any medium, provided the original work is properly cited.

Received: 28 June 2007

Accepted: 23 August 2007

\begin{abstract}
Background: Chronic congestive heart failure (CHF) is a complex disease with rising prevalence, compromised quality of life (QoL), unplanned hospital admissions, high mortality and therefore high burden of illness. The delivery of care for these patients has been criticized and new strategies addressing crucial domains of care have been shown to be effective on patients' health outcomes, although these trials were conducted in secondary care or in highly organised Health Maintenance Organisations. It remains unclear whether a comprehensive primary care-based case management for the treating general practitioner (GP) can improve patients' QoL.
\end{abstract}

Methods/Design: HICMan is a randomised controlled trial with patients as the unit of randomisation. Aim is to evaluate a structured, standardized and comprehensive complex intervention for patients with CHF in a I2-months follow-up trial.

Patients from intervention group receive specific patient leaflets and documentation booklets as well as regular monitoring and screening by a prior trained practice nurse, who gives feedback to the GP upon urgency. Monitoring and screening address aspects of disease-specific selfmanagement, (non)pharmacological adherence and psychosomatic and geriatric comorbidity. GPs are invited to provide a tailored structured counselling 4 times during the trial and receive an additional feedback on pharmacotherapy relevant to prognosis (data of baseline documentation).

Patients from control group receive usual care by their GPs, who were introduced to guidelineoriented management and a tailored health counselling concept.

Main outcome measurement for patients' QoL is the scale physical functioning of the SF-36 health questionnaire in a 12-month follow-up. Secondary outcomes are the disease specific QoL 
measured by the Kansas City Cardiomyopathy questionnaire (KCCQ), depression and anxiety disorders (PHQ-9, GAD-7), adherence (EHFScBS and SANA), quality of care measured by an adapted version of the Patient Chronic Illness Assessment of Care questionnaire (PACIC) and NTproBNP. In addition, comprehensive clinical data are collected about health status, comorbidity, medication and health care utilisation.

Discussion: As the targeted patient group is mostly cared for and treated by GPs, a comprehensive primary care-based guideline implementation including somatic, psychosomatic and organisational aspects of the delivery of care (HICMAn) is a promising intervention applying proven strategies for optimal care.

Trial registration: Current Controlled Trials ISRCTN30822978.

\section{Background}

Congestive Heart Failure (CHF) is a disease with high incidence, prevalence, and the cumulative lifetime risk to develop CHF of 20\% [1-3]. Despite substantial progress in medical treatment [4], one-year mortality ranges stagedependently from 7 to $28 \%$ and increases to $75 \%$ after five years $[5,6]$. The burden of illness includes multiple acute failures followed by hospital admissions [7]. New treatment strategies focus on preventing readmissions and on improving the prognosis. With a higher stage of $\mathrm{CHF}$ the Quality of life (QoL) decreases [8]. Some studies suggest that QoL is a predictor of the course of CHF, independent of the acknowledged somatic predictors of prognosis (like left ventricular ejection fraction) $[8,9]$.

Improvement of mortality rates by pharmacotherapy does not necessarily implicate improvement of QoL[10]. Nonmedical treatment like exercise training and patient education have been shown to have a high impact on QoL [11]. However, it has been shown that the known clinical and somatic predictors explain only about $40 \%$ of the total variance of QoL $[8,12]$.

Transfer of these new insights has not been implemented in daily practice, although specific care taking psychosocial aspects into account has been demanded $[13,14]$. However, there are no concepts for specific training dealing with patients with CHF to improve their QoL in Primary Care. Although QoL is a main concern of patients, it is not a central topic of cardiac research.

To date, only small-scale studies with selective samples analysed data regarding QoL in patients with CHF. In addition, there is no horizontal and vertical networking for transfer of knowledge. This is especially the case for the transfer of specific interventions for general practice. There are several guidelines for the management of patients with heart failure[15], but adherence to these guidelines is low [16].

\section{Novel aspect of integrated case management}

To our knowledge, the proposed study is the first investigating the efficacy of a multifaceted case management in patients with CHF in general practice in Germany. Studies showing the efficacy of case management in patients with CHF were performed in highly organised Health Maintenance Organisations and have therefore limited external validity. GPs in Germany as in many other countries work mostly in single or double practices without multi-professional teams. A nurse specialist exerting a form of structured care is new to the German system. The structured care intervention consists of telephone monitoring, home visits with additional diagnostic screening, medication feedback, patient leaflets, and specific counseling allowing assessment of and intervention in a multitude of aspects of care relevant to the patient. Provided that our intervention proves to be effective, the better treatment of patients with CHF in general practice could prevent personal suffering, improve patient and provider satisfaction, and lower health care costs.

\section{Evidence}

The following studies give evidence for the necessity, clinical relevance, and novelty of the trial: Depressive symptoms and quality of life are strongly associated variables[17]. QoL and depressive disorders are predictive for unscheduled readmissions and mortality[8,9,18-20]. In the "Improvement of Heart Failure Initiative Study" the level of knowledge, the diagnostic as well as the therapeutic approach of 100 GPs (900 patients) have been evaluated. As the results show, patients in Germany are treated according to guidelines only in about $20 \%$ of the cases. Cardiologists and GPs set different priorities concerning diagnostics and therapy $[21,22]$. Improvement of mortality rates by pharmacotherapy does not implicate improvement of QoL[10]. Studies evaluating "complex" case management for $\mathrm{CHF}$ in or starting from secondary care have shown to have favourable outcome with regard to QoL, readmission and mortality [23]. Sustained effects have been shown in long-term follow-ups [24]. 


\section{Methods/Design}

Aims of the study

With the purpose to improve QoL and physical outcomes this study aims to investigate multifaceted case management of patients with CHF in general practice. Therefore, an intervention that specifically addresses the needs of this patient group will be compared to usual treatment as control condition.

\section{Scientific hypothesis}

The case management intervention group shows a significantly better outcome with respect to QoL compared to the control group at the one-year follow-up. We further expect greater improvements in the intervention subjects compared to controls with respect to readmission and mortality, physical outcomes parameters (NYHA and NTproBNP), patients' satisfaction with medical care and health service utilization.

\section{Study/trial design}

A (prospective, ) two-armed, randomised controlled trial will be performed, with the patients as unit of randomisation. This trial design was chosen because of high internal validity, while the number of needed recruited patients remains feasible. Contamination is avoided as the intervention is highly standardized, time-consuming and linked to a personal one-time documentation. Eligible patients will be randomized to either intervention or usual care conditions (see figure 1).

\section{Sample size/Power calculations}

We expect that the differences in QoL between the interventional and control arm to be at least $9 \%$ with superior QoL in the interventional arm (physical functioning, Scale 1 of SF-36). With a serial correlation of $0.6, n=66$ patients are needed per arm for $80 \%$ power at an alphalevel of 5\%. Calculating a drop-out of 30\%, 132 cases represent $70 \%$. Therefore, at least 188 patients will be initially included.

\section{Recruitment of GPs and randomisation}

GPs were eligible for randomization if their practice had a contract with all German insurances, ensuring that patients of all social levels have unlimited admission to the practice. About 200 GPs in the area of Baden-Wuerttemberg, fulfilling the inclusion criteria, were invited by a formal letter from the Department of General Practice and Health Services Research of the University Hospital Heidelberg, to participate in the study. 36 GPs gave their written consent to participate in the study. 5 GPs declined to participate prior to randomisation, two due to lack of eligible patients, two due to high time and effort and one because of disagreement concerning the "evidence based" approach. Based on detailed information about the practice and the GP, the inclusion criteria were checked. No GP or no practice had to be excluded due to the inclusion criteria. Of the participating 31 centres 19 took part in the previous train the trainer-study $(\mathrm{ttt})$ about guideline implementation. In this RCT general practitioners (GPs) of the intervention group got a special training in a clinical practice guideline (CPG) on CHF while GPs of the con-

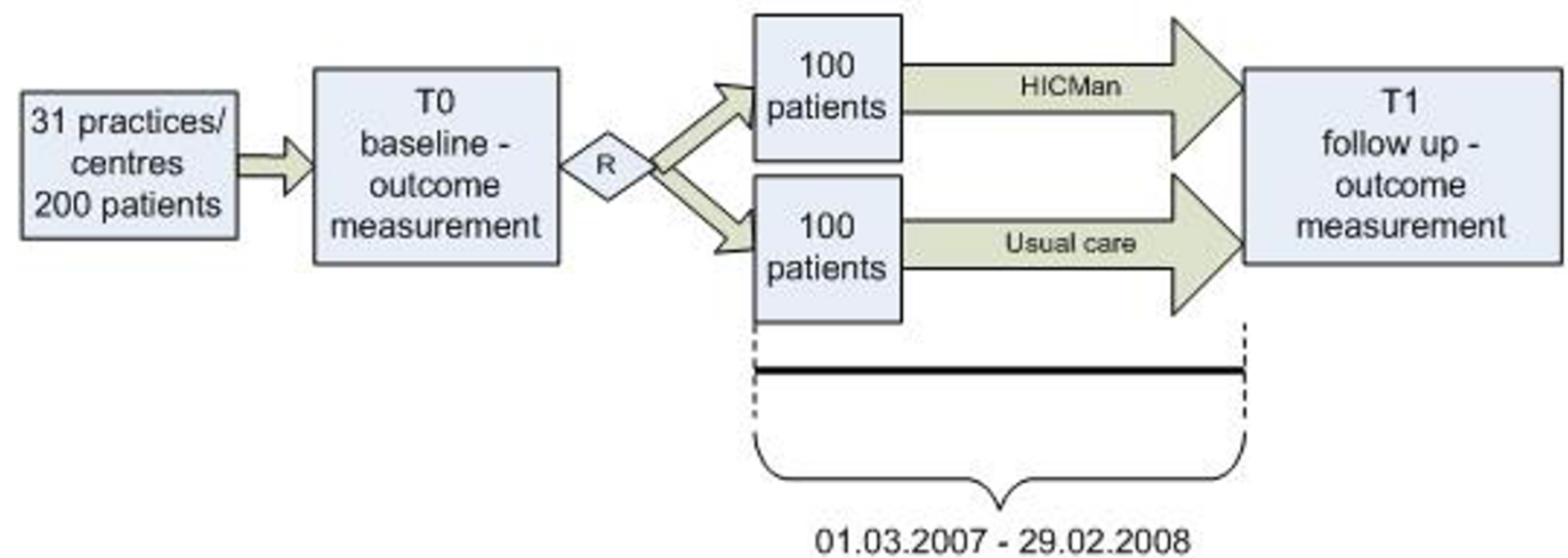

Follow-up: 12 months

\section{Figure I}

Trial design (HICMan-CHF). 
trol group merely got the CPG with a standard lecture, and we compared the QoL of CHF patients in both groups. The participating GPs of the ttt-study were invited to continue in the HICMan-study.

From November 2006 to January 2007, 31 recruiting centres (with about 6-7 recruited patients per centre) enrolled 200 eligible patients and obtained informed consent prior to allocation. We used third party fax randomisation, conducted by the Coordination Centre for Clinical Trials Leipzig (KKSL). KKSL obtained the randomisation faxes directly from the centres and conducted the procedure based on a computer generated, concealed random allocation sequence using an algorithm of Pocock: Patients were grouped by the interval of recruitment time. Each group was randomised immediately at the end of the respective recruitment interval, stratified by arm and centre. Assignment of the patients to their intervention arm was sent by fax from KKSL to the centres. The assessors of outcomes are blinded until post-intervention data collection is completed.

\section{Inclusion/exclusion criteria}

Case manager inclusion criteria

For the practice nurses who wanted to engage in the study, the following criteria had to be fulfilled apart from the participation in the study's case management work shops: status of practice nurse or at least in the third year of formal training.

Patient inclusion criteria

To be eligible for the study, patients had to fulfil the following inclusion criteria: age $>=40$ years, objective left ventricular $\mathrm{CHF}$, ejection fraction of $45 \%$ or less (confirmed by echocardiography within the last 24 months), no dyspnea (NYHA I), but hospital admission because of CHF within the last 24 months or dyspnea NYHA II-IV, stability of the disease at the time of inclusion, capability to give informed consent.

\section{Patient exclusion criteria}

To be eligible for the study, the following criteria had to be excluded: participation in another clinical trial or specific care concept within the last 30 days (incl. telemedicine interventions), residency in a nursing home, primary valvular heart disease with relevant hemodynamic effects, Hypertrophic Obstructive/Restrictive Cardiomyopathy (HOCM/RCM), status post/pre organ transplantation, acute left ventricular failure, short life expectancy of $<2$ years due to serious concomitant illness, known impaired mental state (e.g. dementia) that prevents accurate answers to questions, addictive disorders with continuing drug abuse despite social, legal or professional conflicts.

\section{Data collection}

After giving their informed and written consent to participate in the study, patients were registered in the Centre of the Competence Network of Heart Failure (Berlin) which is responsible for administration, coordination and pseudonymisation of patients' personal data.

Patients will receive a questionnaire for evaluation of generic QoL (SF-36) and disease-specific QoL (KCCQ) as well as other items to assess secondary outcome parameters as shown in table 1. GPs will document clinical data (history, actual clinical status, lab results, ECG etc.) and draw a blood sample for determination of NT-pro-BNP. Analysis of blood samples will be performed by the Charité Universitätsmedizin Berlin. A study nurse will collect the documents in the practice and forward them to the

Table I: Outcome-parameter and instruments

\begin{tabular}{ll}
\hline Outcome-parameter & Instrument \\
\hline $\begin{array}{l}\text { Primary outcome } \\
\text { Quality of life }\end{array}$ & SF-36, scale I (PF) \\
& \\
Secondary outcomes & \\
Quality of life & other scales of SF-36 \\
Disease-specific Quality of life & KCCQ \\
Depression & PHQ-9 \\
Anxiety disorder & GAD-7 \\
Patient assessment of care & Modified PACIC \\
Adherence & EHFScBS and SANA \\
Disease course & NT-proBNP \\
& \\
Other comorbidity & CIRS, small geriatric assessment \\
Health care utilisation & Case report form (CRF) \\
Medication & CRF \\
Sociodemographic variables & Questionnaire \\
HICMan documentation of CM & CRF, PHQ-9, GAD-7 and small geriatric assessment \\
\hline
\end{tabular}


Coordination Centre for Clinical Trials Leipzig (KKSL, Germany), which is responsible for data management (database set-up and validation, data entry, coding, query management and case report form printing).

\section{Outcome-parameter}

\section{Primary and secondary outcomes}

Table 1 displays the outcome parameters and associated instruments used. The primary outcome is quality of life assessed by the Short Form 36 Health Questionnaire (physical function scale)[25], an internationally validated generic instrument for the assessment of quality of life.

Secondary outcomes are the other scales of SF-36[25], disease-specific QoL (KCCQ [26]), signs for depressive and anxiety disorders (PHQ-9 and GAD-7)[27,28], Patient Assessment of Chronic Illness Care (modified PACIC [29]), admission to hospital or death due to heart failure (combined), improvement of heart failure according to functional status (NYHA) and NT-proBNP [30], the European Heart Failure Selfcare Behaviour Scale (EHFScBS[31]), the Scales to Assess Low Adherence (SANA[32]), evidence-based pharmacotherapy and costeffectiveness. Additionally, the case management will be analysed. This includes the used monitoring list and screening tools (study specific assessment of patients behaviour regarding life style, PHQ-9, GAD-7 and small geriatric assessment [33-35]).

These data will be compiled from patient questionnaires and case report forms of the patients. All instruments except SANA and PACIC represent well established instruments that are validated in Germany. No interim analysis will be performed except for pharmacotherapy to provide prescription feedback.

\section{Intervention/Follow-ups}

Patients randomised to the intervention arm will get case management care by a specifically trained practice nurse (case manager $=\mathrm{CM}$ ). Duration of training was 1,5 days.

All patients receive an introduction of the $\mathrm{CM}$, monitoring by telephone, home visits, disease-specific counselling, and surveillance concerning prescription. Details are listed below:

1. Introduction: After enrolment, the $\mathrm{CM}$ will take approximately $30 \mathrm{~min}$. for introducing her function and establishing a relationship to the patient. Patients get information about their disease, realisation of symptoms and self-monitoring (shortness of breath, fatigue, peripheral oedema, weight, heart rate and blood pressure). On that occasion, patients will get an information booklet and documentation booklet.
2. Monitoring by telephone: The CM carries out a telephone monitoring according to the patients' risk:

- low to medium risk (NYHA I/II) - every six weeks, three personal home visits during the year instead of telephone monitoring.

- high risk (NYHA III/IV) - every three weeks, three personal home visits during the year instead of telephone monitoring.

Content of the telephone interview will check for physical warning physical signs, problems with medication (adherence).

3. Home visits: During home visits (three times a year) certain domains from the following will be evaluated in a structured and operated way tailored to heart failure patients: Physical, cardiac status; structured assessment of lifestyle [36] (as first step part of a 5-A counselling, see below); adherence; depression and anxiety disorder screening $(2 \times)$; small geriatric assessment $(2 x)$ and a detailed medication check $(1 \times)$.

4. After the introduction and the three home visits, the patients will get a specific physician encounter for counselling as part of a 5-A counselling $[37,38]$ based on the structured assessment of relevant lifestyle and habits in relation to heart failure concerning the topics (i) self management, (ii) physical activity and (iii) smoking.

5. Recall-Reminder-Systems: if necessary, active surveillance concerning prescription or doctor follow-ups will be applied.

6. In month seven, GPs will receive an additional feedback on pharmacotherapy relevant to prognosis for patients from the IG (data of baseline documentation). Substances from the following classes will be transformed from the following three drug classes: ACE-inhibitors (or angiotensin-II receptor antagonists), beta-blockers and aldosterone antagonists. Percentage of reached target dose (from current guideline) will be calculated. The procedure will be performed by a statistician not involved in the conduct of the study. GPs will get a printout showing a graphical depiction of each patient from the IG (he is caring for).

\section{Actions to take by the $C M$}

During each visit, the CM will apply a structured and formalised assessment of cardiac/physical functioning and additional screening according to the structured plan, as outlined above. Results of the screening will guide the CM to tangible actions, which consist of a feedback or, if nec- 
essary, of direct contact to/with the treating physician upon urgency.

\section{Control condition}

Participating physicians will receive a guideline for the management of heart failure as the recommended standard and the introduction to a structured counselling for heart failure (5-A). No case management will be applied to the patients of this group.

\section{Follow-ups}

$\mathrm{CM}$ home visits will take place at the beginning, after the first and the second third of the year. Assessment and documentation will take place at the beginning and the end of the year (see figure 1).

\section{Timeframe of the study}

The study has the following phases: Training of study nurse and preparation of trial (months $1-6)$, recruitment (months 1-6), treatment/control (months $7-18$ ), follow-up (month 18), data entry (months 19-20), data analyses and publication of results (months 21-24). At the time of drafting the manuscript, the planned time schedule is kept: 200 patients were enrolled. The study was in the phase of treatment/control in month 10.

\section{Description of risks}

Serious risks or undesired effects of questionnaires have not been described in the literature. No specific risk for participating patients can be stated. The management of the patients remains in the responsibility of the treating physician.

\section{Ethical and legal aspects Ethical principles}

The study is being conducted in accordance with medical professional codex and the Helsinki Declaration, i.e. the ICH Guideline for Good Clinical Practice (GCP) E6 of 1996, the CPMP/ICH/135/95 of September 1997 and its amendments [39-41]. The study is also in accordance with the German Federal Data Security Law (BDSG). All professionals participating at the study oblige themselves to adhere to the abovementioned declaration and law.

Study participation of patients is voluntary and can be cancelled at any time without provision of reasons and without negative consequences for their future medical care.

\section{Patient informed consent}

Previous to study participation patients receive written and spoken information about the content and extent of the planned study; for instance about potential benefits for their health and potential risks. In case of acceptance they sign the informed consent form. In case of study dis- continuation all material will be destroyed or the patient will be asked if he/she accepts that existing material can be analysed in the study.

\section{Legal principles}

Vote of the ethics committee

The study protocol was approved by the ethics committee of the University of Heidelberg previous to the start of the study in November 2006. Inclusion of patients/participants did not start unless there was a written and unrestricted positive vote of the ethics committee (approval number 303/2006). The study protocol was also approved by the ethics committee of the medical association of the state of Baden-Württemberg (approval number B-244-06-f). Both votes were received in October 2006. The study is registered (ISRCTN30822978).

\section{Data security/disclosure of original documents}

The patient names and all other confidential information fall under medical confidentiality rules and are treated according to German Federal Data Security Law (BDSG). The patient questionnaires and original CRFs are collected by the study nurse and mailed to the KKSL. These study related data and documents are stored on a protected central server of the KKSL. Only direct members of the internal study team can access the respective files.

Intermediate and final reports as well as the documentation of the Case management are stored in the office of the Department of General Practice and Health Services Research at the University Hospital Heidelberg.

The press copies of the clinical data and the documentation of the case management are stored in the centres for at least 10 years.

\section{Discussion}

As the targeted patient group is mostly cared for and treated by GPs, a comprehensive primary care-based guideline implementation including somatic, psychosomatic and organisational aspects of the delivery of care (HICMAn) is a promising intervention applying proven strategies for optimal care of patients with CHF.

\section{Competing interests}

The author(s) declare that they have no competing interests.

\section{Authors' contributions}

FPK, TMT, AB, DS, WH and JS conceived and performed the study. FPK, TMT, JG and CM developed the intervention. All authors contributed to, read and approved the final manuscript. 


\section{Acknowledgements}

This study is part of the project "Quality of life" (No. 10 of the Competence Network of Heart Failure) that aims to improve the quality of life of patients suffering from CHF. The project is financed by the German Federal Ministry of Education and Research (BMBF), grant-number 0I GI0205/2I. We are indebted to Annika Barth and staff members for essential and prompt contributions to the production of the investigator files. The following trial-supporting facilities of the Competence Network of Heart Failure are involved as outlined above:

I. Centre of the Compentence Network of Heart Failure (Berlin): Administration, coordination and pseudonymisation of patients' personal data: Kompetenznetz Herzinsuffizienz, Augustenburger Platz I, I3353 Berlin, Germany, Tel: 030/450 576 8I2, Fax: 030/450 576962.

2. Data management, IT (e.g. database set-up and validation, data entry, coding, query management) and case report form (CRF), CRF printing: Coordination Centre for Clinical Trails Leipzig (KKSL), Härtelstr. 16-18, 04107 Leipzig, Germany.

3. Analysis of blood samples: BNP, Charité - Universitätsmedizin Berlin, Campi Virchow-Klinikum und Buch, Medizinische Klinik mit Schwerpunkt Kardiologie, Augustenburger Platz I, 13353.

\section{References}

I. McMurray JJ, Stewart S: Epidemiology, aetiology, and prognosis of heart failure. Heart 2000, 83:596-602.

2. Bursi F, Weston SA, Redfield MM, Jacobsen SJ, Pakhomov S, Nkomo VT, Meverden RA, Roger VL: Systolic and diastolic heart failure in the community. JAMA 2006, 296:2209-2216.

3. Fischer M, Baessler A, Holmer SR, Muscholl M, Brockel U, Luchner A, Hense HW, Doring A, Riegger G, Schunkert H: [Epidemiology of left ventricular systolic dysfunction in the general population of Germany: results of an echocardiographic study of a large population-based sample]. Z Kardiol 2003, 92:294-302.

4. Shekelle PG, Rich MW, Morton SC, Atkinson CS, Tu W, Maglione M, Rhodes S, Barrett M, Fonarow GC, Greenberg B, Heidenreich PA, Knabel T, Konstam MA, Steimle A, Warner SL: Efficacy of angiotensin-converting enzyme inhibitors and beta-blockers in the management of left ventricular systolic dysfunction according to race, gender, and diabetic status: a meta-analysis of major clinical trials. J Am Coll Cardiol 2003, 4I: I529- 1538.

5. Stewart S, Maclntyre K, Hole DJ, Capewell S, McMurray JJ: More 'malignant' than cancer? Five-year survival following a first admission for heart failure. Eur J Heart Fail 200I, 3:3I5-322.

6. Muntwyler J, Abetel G, Gruner C, Follath F: One-year mortality among unselected outpatients with heart failure. Eur Heart J 2002, 23: $|86|-\mid 866$

7. Opasich C, Rapezzi C, Lucci D, Gorini M, Pozzar F, Zanelli E, Tavazzi L, Maggioni AP: Precipitating factors and decision-making processes of short-term worsening heart failure despite "optimal" treatment (from the IN-CHF Registry). Am J Cardiol 200I, 88:382-387.

8. Juenger J, Schellberg D, Kraemer S, Haunstetter A, Zugck C, Herzog $W$, Haass M: Health related quality of life in patients with congestive heart failure: comparison with other chronic diseases and relation to functional variables. Heart 2002, 87:235-24I.

9. Konstam V, Salem D, Pouleur H, Kostis J, Gorkin L, Shumaker S, Mottard I, Woods P, Konstam MA, Yusuf S: Baseline quality of life as a predictor of mortality and hospitalization in 5,025 patients with congestive heart failure. SOLVD Investigations. Studies of Left Ventricular Dysfunction Investigators. Am J Cardiol 1996, 78:890-895.

10. Cohn JN, Tognoni G: A randomized trial of the angiotensinreceptor blocker valsartan in chronic heart failure. $N$ Engl J Med 200I, 345:1667-1675.

II. Grady KL, Dracup K, Kennedy G, Moser DK, Piano M, Warner Stevenson $L$, Young JB: Team management of patients with heart failure. Circulation 2000, 102:2443-2456.
12. Steptoe A, Mohabir A, Mahon NG, McKenna W]: Health related quality of life and psychological wellbeing in patients with dilated cardiomyopathy. Heart 2000, 83:645-650.

13. Moser DK, Mann DL: Improving outcomes in heart failure: It's not unusual beyond usual care. Circulation 2002, 105:28I0-28I2.

14. Smith JJ, Konstam MA: Heart failure: A case for subspecialized care management. Am Heart J 1999, 138: |4-16.

15. Remme W], Swedberg K: Guidelines for the diagnosis and treatment of chronic heart failure. Eur Heart J 200I, 22: I527-I560.

16. Cleland JG, Cohen-Solal A, Aguilar JC, Dietz R, Eastaugh J, Follath F, Freemantle N, Gavazzi A, van Gilst WH, Hobbs FD, Korewicki J, Madeira HC, Preda I, Swedberg K, Widimsky J: Management of heart failure in primary care (the IMPROVEMENT of Heart Failure Programme): an international survey. Lancet 2002, 360:1631-1639.

17. Ruo B, Rumsfeld JS, Hlatky MA, Liu H, Browner WS, Whooley MA: Depressive symptoms and health-related quality of life: the Heart and Soul Study. JAMA 2003, 290:215-22I.

18. Bouvy ML, Heerdink ER, Leufkens HG, Hoes AW: Predicting mortality in patients with heart failure: a pragmatic approach. Heart 2003, 89:605-609.

19. Jaagosild P, Dawson NV, Thomas C, Wenger NS, Tsevat J, Knaus WA, Califf RM, Goldman L, Vidaillet H, Connors AF Jr.: Outcomes of acute exacerbation of severe congestive heart failure: quality of life, resource use, and survival. SUPPORT Investigators. The Study to Understand Prognosis and Preferences for Outcomes and Risks of Treatments. Arch Intern Med 1998, 158:108I-1089.

20. Jiang W, Alexander J, Christopher E, Kuchibhatla M, Gaulden LH, Cuffe MS, Blazing MA, Davenport C, Califf RM, Krishnan RR, O'Connor CM: Relationship of depression to increased risk of mortality and rehospitalization in patients with congestive heart failure. Arch Intern Med 200I, 161:1849-1856.

21. Rutten FH, Grobbee DE, Hoes AW: Diagnosis and management of heart failure: a questionnaire among general practitioners and cardiologists. Eur J Heart Fail 2003, 5:345-348.

22. Rutten $\mathrm{FH}$, Grobbee DE, Hoes AW: Differences between genera practitioners and cardiologists in diagnosis and management of heart failure: a survey in every-day practice. Eur J Heart Fail 2003, 5:337-344.

23. Holland R, Battersby J, Harvey I, Lenaghan E, Smith J, Hay L: Systematic review of multidisciplinary interventions in heart failure. Heart 2005, $91: 899-906$.

24. Stewart S, Horowitz JD: Home-based intervention in congestive heart failure: long-term implications on readmission and survival. Circulation 2002, 105:2861-2866.

25. Bullinger M, Kirchberger I, Ware J: Der deutsche SF-36 Health Survey. Z f Gesundheitswiss 1995, I:21-36.

26. Green CP, Porter CB, Brensnahan DR, Spertus JA: KCCQ/ Kansas City Cardiomyopathy Questionnaire 2000.

27. Löwe B, Spitzer RL, Zipfel S, W H: PHQ-D Gesundheitsfragebogen für Patienten. Pfizer, Karlsruhe 200I.

28. Spitzer RL, Kroenke K, Williams JB, Lowe B: A brief measure for assessing generalized anxiety disorder: the GAD-7. Arch Intern Med 2006, 166:1092-1097.

29. Glasgow RE, Wagner EH, Schaefer J, Mahoney LD, Reid RJ, Greene SM: Development and validation of the Patient Assessment of Chronic Illness Care (PACIC). Med Care 2005, 43:436-444.

30. Richards M, Troughton RW: NT-proBNP in heart failure: therapy decisions and monitoring. Eur J Heart Fail 2004, 6:35I-354.

31. Jaarsma T, Strömberg A, Martensson J, Dracup K: Development and testing of the European Heart Failure Self-Care Behaviour Scale. The European Journal of Heart Failure 2003, 5:363-370.

32. Mathe C, Schellberg D: SANA/ Skala zur Einschätzung niedriger Adherence 2004.

33. Kalbe E, Kessler J, Calabrese P, Smith R, Passmore AP, Brand M, Bullock R: DemTect: a new, sensitive cognitive screening test to support the diagnosis of mild cognitive impairment and early dementia. Int J Geriatr Psychiatry 2004, 19:136-143.

34. Gulich M, Zeitler HP: [The walking-counting test. A simple test for assessing the risk of falling]. Dtsch Med Wochenschr 2000, 125:245-248.

35. MAHONEY FI, BARTHEL DW: FUNCTIONAL EVALUATION: THE BARTHEL INDEX. Md State Med J 1965, 14:61-65.

36. Prochaska JO, Velicer WF: The transtheoretical model of health behavior change. Am J Health Promot 1997, 12:38-48. 
37. Glasgow RE, Davis CL, Funnell MM, Beck A: Implementing practical interventions to support chronic illness self-management. Jt Comm J Qual Saf 2003, 29:563-574.

38. Glasgow RE, Funnell MM, Bonomi AE, Davis C, Beckham V, Wagner EH: Self-management aspects of the improving chronic illness care breakthrough series: implementation with diabetes and heart failure teams. Ann Behav Med 2002, 24:80-87.

39. Declaration of Helsinki: Guiding Physicians in Biomedical Research Involving Human Subjects. Adopted by the 18th World Medical Assembly, Helsinki (Finland), June 1964. Last amendment by the 48th General Assembly, Somerset West (Rep. of South Africa). 1996.

40. International Conference on Harmonisation of Technical Requirements for the Registration of Pharmaceutical Products for Human Use: ICH Harmonized Tripartite Guideline, "Guideline for Good Clinical Practice". Recommended for Adoption at Step 4 of the ICH Process on I May 19961996 [http://www.ifpma.org/ich5e.htm|\#GCP].

41. International Conference on Harmonisation of Technical Requirements for the Registration of Pharmaceutical Products for Human Use: ICH Harmonized Tripartite Guideline, "Clinical Data Safety Management: Definitions and Standards for Expedited Reporting". Recommended for Adoption at Step 4 of the ICH Process on 27 May 1994 I994 [http:// www.ifpma.org/ich5e.html\#Safety].

\section{Pre-publication history}

The pre-publication history for this paper can be accessed here:

http://www.biomedcentral.com/1471-2261/7/25/prepub

Publish with Bio Med Central and every scientist can read your work free of charge

"BioMed Central will be the most significant development for disseminating the results of biomedical research in our lifetime. "

Sir Paul Nurse, Cancer Research UK

Your research papers will be:

- available free of charge to the entire biomedical community

- peer reviewed and published immediately upon acceptance

- cited in PubMed and archived on PubMed Central

- yours - you keep the copyright
BioMedcentral 